\title{
An efficient multi-standard QC-LDPC decoder based on the row-layered decoding algorithm
}

\author{
Song Guo ${ }^{a)}$, Yong Dou, Yuanwu Lei, Rongchun Li, and Yu Li \\ National Laboratory for Parallel and Distributed Processing, \\ National University of Defense Technology, Changsha, China \\ a)songguo@nudt.edu.cn
}

\begin{abstract}
This paper presents an efficient multi-standard decoder for quasicyclic low-density parity-check (QC-LDPC) codes. By employing the configurable multi-bank memory structure, simplified permutation network and multiple pipelined datapaths, the decoder can efficiently support WiMAX and WiFi standards without any structural changes. The offset-min-sumbased row-layered decoding algorithm is adopted to reduce the computation complexity. Based on these optimizations, the proposed decoder can achieve about $658 \mathrm{Mbps}$ (1410 Mbps) and $527 \mathrm{Mbps}(1129 \mathrm{Mbps})$ for WiMAX and WiFi standards on TSMC $130 \mathrm{~nm}$ (and $65 \mathrm{~nm}$ ) Application Specific Integrated Circuits (ASICs) platforms, respectively. Compared with the previous works, the throughput can obtain up to $10 \times$ improvement for WiMAX standard and $5 \times$ improvement for WiFi standard.
\end{abstract}

Keywords: multi-standard decoder, QC-LDPC, multi-bank memory structure, offset-min-sum-based row-layered decoding

Classification: Electron devices, circuits, and systems

\section{References}

[1] R. Gallager: IRE Trans. Inf. Theory 8 (1962) 21. DOI:10.1109/TIT.1962. 1057683

[2] D. MacKay and R. Neal: Electron. Lett. 18 (1996). DOI:10.1049/el:19961141

[3] G. Gentile, M. Rovini and L. Fanucci: ISTC (2010) 294. DOI:10.1109/ISTC. 2010.5613886

[4] C.-H. Liu, C.-C. Lin, S.-W. Yen, C.-L. Chen, H.-C. Chang, C.-Y. Lee, Y.-S. Hsu and S.-J. Jou: IEEE Trans. Circuits Syst. II, Exp. Briefs 56 (2009) 734. DOI:10. 1109/TCSII.2009.2027967

[5] M. Awais, A. Singh, E. Boutillon and G. Masera: DSD (2011) 340. DOI:10. 1109/DSD.2011.112

[6] Y. Huang, C. Chen, C. Zhou, Y. Chen and X. Zeng: ISOCC (2011) 54. DOI:10. 1109/ISOCC.2011.6138644

[7] C. Roth, P. Meinerzhagen, C. Studer and A. Burg: A-SSCC (2010). DOI:10. 1109/ASSCC.2010.5716618

[8] G. Tzimpragos, C. Kachris, D. Soudris and I. Tomkos: IPDPSW2014 (2014) 269. DOI:10.1109/IPDPSW.2014.36 
[9] B. Xiang, D. Bo, S. Huang and X. Zeng: IEEE J. Solid-State Circuits 46 (2011) 1416. DOI: $10.1109 / \mathrm{JSSC} .2011 .2125030$

\section{Introduction}

The low-density parity-check (LDPC) codes, proposed by Robert Gallager in 1962 [1] and rediscovered by MacKay and Neal in 1996 [2], have been considered as one of the most promising near-optimal error correcting codes, due to its powerful error-correction capabilities and high throughput. QC-LDPC codes, one kind of the most important LDPC codes, have been widely used in many industrial standards, such as WiMAX, WiFi, CMMB, and so on.

It is essential to efficiently support multi-standard LDPC codes to make the use of the real products convenience. However, because of variable code lengths and block sizes of different standards, it is a challenging topic to design a single decoder to efficiently support the decoding of multi-standard QC-LDPC codes. Some studies have attempted to implement the multi-standard decoders on ASIC. Giuseppe Gentile, et al. proposed a SIMD-based decoder on ASIC to support WiMAX and WiFi standards by means of the shared memory [3], however, the implementation of the memory management unit is a challenge. Chih-Hao Liu, et al. implemented a multimode decoder with a reconfigurable message-passing network to support WiMAX and WiFi standards [4], however, the performance degradation resulted from the complex permutation network. M. Awais, et al. employed a fully parallel check node and block level channel memory organization scheme to design a multi-standard decoder [5]. Yuebing Huang, et al. exploited a common architecture with the shared memory and logic devices to support LDPC and Turbo codes [6]. However, these two designs cannot dynamically support different standards.

In this paper, we present an efficient multi-standard decoder for QC-LDPC codes. By exploiting the configurable multi-bank memory structure and simplified permutation network, the decoder can support WiMAX and WiFi standards without any architectural changes. The offset-min-sum-based row-layered decoding algorithm is adopted to reduce the computation complexity. Meanwhile, multiple pipelined datapaths are employed to process multiple row layers in parallel and reduce the latency of each row layer. Based on the above techniques, the proposed decoder can achieve $658 \mathrm{Mbps}(1410 \mathrm{Mbps})$ and $527 \mathrm{Mbps}$ (1129 Mbps) for WiMAX and WiFi standards using TSMC $130 \mathrm{~nm}$ (or $65 \mathrm{~nm}$ ) technology, respectively. Compared with the previous works, the throughput can obtain up to $10 \times$ improvement for WiMAX standard and $5 \times$ improvement for WiFi standard.

The rest of this paper is organized as follows. Section 2 gives a brief overview of LDPC codes and the row-layered decoding algorithm. The overall architecture of the proposed decoder is illustrated in Section 3. The performance of the decoder is evaluated and compared in Section 4. Finally, the main conclusion of this paper is drawn in Section 5. 


\section{QC-LDPC codes and decoding algorithm}

\subsection{Quasi-cyclic low-density parity-check code}

A binary QC-LDPC code is a linear block code specified by a sparse parity-check matrix $H, H x^{T}=0$, where $x$ is the codeword. $H$ consists of $M_{b} \times N_{b}$ square submatrices. Each sub-matrix is either a $Z \times Z$ zero matrix or circulant matrix, where $Z$ is the expansion factor and can be calculated as $Z=N / N_{b}, N$ representing the column number of $H$. Table I lists the main parameters of WiMAX and WiFi standards used in this paper, where the code length (576:96:2304) means that the code length ranges from 576 to 2304 with the step of 96 .

Table I. Parameters of the WiMAX and WiFi standards

\begin{tabular}{|c|c|c|c|c|c|}
\hline Standard & Code Length & code rate & Block Size & $N_{b}$ & $M_{b}$ \\
\hline WiMAX & $576: 96: 2304$ & $\begin{array}{c}1 / 2,2 / 3(\mathrm{~A}, \mathrm{~B}), \\
3 / 4(\mathrm{~A}, \mathrm{~B}), 5 / 6\end{array}$ & $24: 4: 96$ & 24 & $4,6,8,12$ \\
\hline WiFi & $648: 648: 1944$ & $1 / 2,2 / 3,3 / 4,5 / 6$ & $27: 27: 81$ & 24 & $4,6,8,12$ \\
\hline
\end{tabular}

\subsection{Offset min-sum based row-layered decoding algorithm}

The row-layered (RL) decoding algorithm is proposed by Mansour and Shanbhag [7]. The RL decoding algorithm is an iteration process controlled by the maximum iteration number. When the decoding process finishes, the final decoded codes are obtained according to the Log-Likelihood Ratio ( $L L R)$ message of each variable node $(V N)$, corresponding to one column of $H$. In each sub-iteration, the check nodes $(C N)$, each corresponding to one row of $H$, are divided into subgroups called layers, and messages are updated layer by layer. In order to reduce the computational complexity, the offset min-sum algorithm is adopted in the sub-iteration.

Taking the $j$-th layer in the $i$-th iteration as an example, for each $\mathrm{VN} v$, the variable-to-check (VTC) message $L_{v c}^{i, j}$ can be gained by subtracting the old checkto-variable (CTV) message from the $L L R$ as Equation (1). As Equation (2), the CTV message $R_{c v}^{i, j}$ is the minimum among the VTC messages received by the CN $c$, where $N(c) \backslash v$ means the set of VNs connected to $c$, except $v$. A compensation factor is multiplied to improve the bit-error-rate performance. Finally, the updated LLR messages for VNs can be obtained by adding the VTC messages and CTV messages as Equation (3).

$$
\begin{gathered}
L_{v c}^{n e w, l}=L_{v}^{n e w,(l-1)}-R_{c v}^{o l d, l} \\
R_{c v}^{n e w, l}=\alpha \times \prod_{n \in N(c) \backslash v} \operatorname{sign}\left(L_{n c}^{n e w}, l\right) \times \min _{n \in N(c) \backslash v}\left|L_{n c}^{\text {new }, l}\right| \\
L_{v}^{n e w, l}=L_{v c}^{n e w, l}+R_{c v}^{\text {new }, l}
\end{gathered}
$$

\section{Architecture of the multi-standard QC-LDPC decoder}

\subsection{Overall architecture of the decoder}

As shown in Fig. 1, the proposed decoder mainly consists of four components, Control Unit, Message Memory, Processing Element Array and Decoding Unit. The control Unit reads the code parameters listed in Table I and circulant offsets of the nonzero sub-blocks to configure the decoder. By reading the information of 


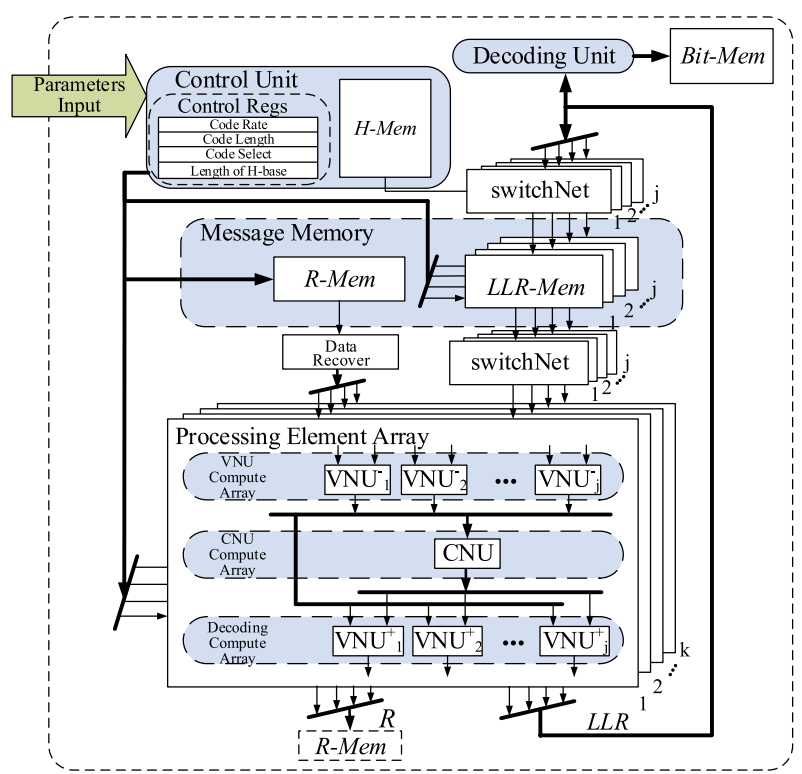

Fig. 1. Overall structure of the proposed decoder

different modes, the decoder can support WiMAX and WiFi standards without any structural changes. The Message Memory, consisting of R-Mem and LLR-Mem, is used to store the CTV messages $R$ and the channel output soft messages $L L R$ for each bit of the codeword. The R-Mem is initialized to be zero, and the LLR-Mem is initialized with the initial $L L R$ messages.

After the initialization of the decoder, the messages $L L R$ and $R$ are read and fed into the Processing Element Array to perform the offset-min-sum-based rowlayered decoding algorithm. When the maximum iteration number is reached, the messages $L L R$ are fed into the Decoding Unit to gain the decoded codeword, and finally, the codeword is stored into the Bit-Mem.

\subsection{Structure of the Message Memory}

The Message Memory, consisting of LLR-Mem and R-Mem, is used to store the intermediate messages during the iterations of the decoding. There are two important features with the proposed Message Memory. The first one is that it can be configured to support variable code lengths and block sizes. The second one is that it can keep the "one cycle multiple layers accesses" time schedule to gain higher decoding throughput.

As shown in Fig. 2, the LLR-Mem adopts a two-level memory structure to realize the flexibility and high parallelism.

In the first level, as there are 24 sub-blocks in one block row of $H$ in both standards, the $L L R-M e m$ is divided into 24 banks to support arbitrary row weights of both standards. Each bank is used to store the $L L R$ messages of one sub-block.

In the second level, according to the increasing steps in the blocks sizes of the WiMAX standard, each bank consists of four linearly-addressed sub-banks, and the width of each sub-bank is $W$ bits, where $W$ is the quantization bits of the $L L R$ messages. In the WiMAX standard, due to the maximum block size being 96, the maximum depth of each sub-bank is 24 . While in the WiFi standard, the block size cannot be divided by four. In order to be compatible with these two standards, 


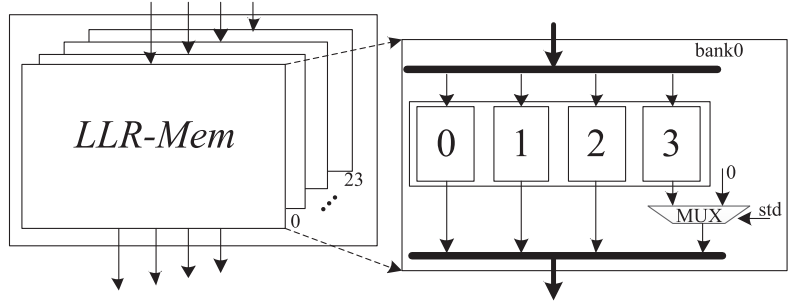

Fig. 2. Overall structure of the LLR-Mem

one flag std is introduced to mask the fourth bank, where the flag std is set to be false to indicate that the fourth bank is invalid, otherwise not. The maximum depth of each sub-bank of the former three sub-banks is 27 .

Based on the above discussion, the maximum depth of both standards is chosen to support both standards. The actual depth of each bank is $B S / 4$ for WiMAX standard, and $B S / 3$ for WiFi standard, where $B S$ represents the sizes of the subblocks of different modes.

Under this structure, the permutation network switchNet can be implemented as follows. In the WiMAX standard, for a given $L L R$ message with address $a d d r$, the least significant two bits of $a d d r$ are used to find the right sub-bank, and the left bits are used as the address in the sub-bank. While in the WiFi standard, the quotient of $a d d r$ and three is used to find the right sub-bank, and remainder is the address in the sub-bank.

The $R$-Mem is used to store the CTV messages $R$. In the QC-LDPC codes, because no overlapped nonzero elements among all the rows in one block row, the corresponding check nodes can be simultaneously updated in parallel. Given the parallelism degree of the LLR-Mem, one four-word compression scheme is adopted in the $R$-Mem, where each word contains the first two minima, the index of the first minimum and the signs of all the VTC messages corresponding to each check node.

\subsection{Structure of the processing element array}

In the QC-LDPC codes, because there are no overlapped nonzero elements among the rows in one block row, these rows can be processed in parallel. Given the parallelism of the Message Memory, four parallel pipelined datapaths are employed to process four row layers in parallel.

Corresponding to three steps in the row-layered decoding algorithm, each datapath mainly consists of three parts, VNU Compute Array (VCA), CNU Compute Array (CCA) and Decoding Compute Array (DCA). In order to support different row weights of WiMAX and WiFi standards, twenty-four VNUs are employed in the VCA, where each VNU corresponds to one sub-block in one block row. Each VNU only consists of one pipelined adder to calculate the VTC messages as Equation (1). In the CCA, the CNU is used to perform the Equation (2) to compute the first two minima and the index of the first minimum among the VTC messages for the corresponding check node as the approach in [8]. And the CTV messages of four check nodes are written back into the $R$-Mem in the fourword compression scheme. As Equation (3), the LLR messages of variable nodes are gained by the addition of the updated CTV messages $R$ and the VTC messages $L_{v c}$ in the DCA, and stored into the LLR-Mem through the switchNet. 


\section{Performance evaluation and comparison}

For the performance evaluation and comparison, the proposed multi-standard decoder is implemented on TSMC $130 \mathrm{~nm}$ and $65 \mathrm{~nm}$ ASIC platforms. Table II summarizes the results of the performance comparison with the previous works. It can be seen that the proposed decoder can achieve the highest throughput, $658 \mathrm{Mbps}$ (1410 Mbps) for WiMAX standard and $527 \mathrm{Mbps}$ (1129 Mbps) for WiFi standard on TSMC $130 \mathrm{~nm}$ (and $65 \mathrm{~nm}$ ) ASIC platforms, respectively.

Compared with the works capable of supporting multiple standards $[3,5,6]$, the throughput (TP) can gain up to about $10 \times$ improvement, and the ratio of throughput and area (TAR) can be improved up to about $25 \times$. These improvements mainly result from the simple memory structure and permutation network, which can increase the frequency of the decoder and exploit the parallelism of the decoding process to improve the throughput of the decoder. Compared with the works only supporting one standard [7,9], the proposed decoder has a little performance loss in terms of the TP and TAR. The reason is that the structural complexity of these standard-specific decoders can be minimized.

Table II. Performance comparison of different platforms

\begin{tabular}{|c|c|c|c|c|c|c|}
\hline Ref. & Our Work & {$[3]$} & {$[5]$} & {$[6]$} & {$[7]$} & {$[9]$} \\
\hline Tech. $(\mathrm{nm})$ & $130 / 65$ & 45 & 130 & 130 & 90 & 130 \\
\hline Standard & Both & Both & Both & Both & Single & Single \\
\hline Width & 7 & $5 / 7$ & 7 & $5 / 6$ & 5 & 6 \\
\hline Freq. & $666.7 / 1428.6$ & 300 & 150 & 160 & 346 & 214 \\
\hline Area & $2.95 / 1.11$ & 0.9 & $2.76 / 2.74$ & 2.8 & 1.77 & 3.03 \\
\hline TP $($ Mbps) & $658 / 1410$ & 122.8 & $240 / 249$ & 136 & 679 & 955 \\
\hline TAR & $223.1 / 1270.3$ & 136.4 & $86.8 / 90.7$ & 48.6 & 383.6 & 315.2 \\
\hline
\end{tabular}

\section{Conclusion}

In this paper, an efficient multi-standard decoder for QC-LDPC codes is presented. By employing the configurable multi-bank memory structure, simplified permutation network, and multiple pipelined datapaths, the proposed decoder can efficiently support WiMAX and WiFi standards without any structural changes. The offsetmin-sum-based row-layered decoding algorithm is adopted in the decoder to reduce the computational complexity. Based on these techniques, the multi-standard decoder can achieve $658 \mathrm{Mbps}(1410 \mathrm{Mbps})$ and $527 \mathrm{Mbps}$ (1129 Mbps) for WiMAX and WiFi on TSMC $130 \mathrm{~nm}$ (and $65 \mathrm{~nm}$ ) ASIC platforms, respectively. Compared with the previous works, the throughput can gain about $10 \times$ improvement for WiMAX and $5 \times$ improvement for WiFi, respectively.

\section{Acknowledgments}

This work is supported by the National High Technology Research and Development Program of China under No. 2012AA012706 and the National Science Foundation of China under Grants of 61402499. 